\title{
Dependence structure analysis with copula GARCH method and for data set suitable copula selection
}

\author{
Ayse Metin Karakas ${ }^{1 *}$
}

\begin{abstract}
Objective: Multivariate GARCH (MGARCH) models are forecasted under normality. In this study, for nonelliptically distributed the data set which are generated Weilbull distribution. Copula-based GARCH (CopulaGARCH) was used. The aim of the paper is to model GARCH for non-normal distributions using copulas.

Material and Methods: A two-step Copula-GARCH model to analyze the dependence structure of data sets was used. In the first step, we show data using univariate GARCH model to get standard residuals and construct marginal distributions. In this section GARCH $(\mathrm{p}, \mathrm{q})$ and $\operatorname{GARCH}(1,1)$ method are introduced. GARCH $(1,1)$ method for data set was used for first step. In the second step, for dependence structures of the data sets were calculated Kendall Tau and Spearman Rho values which are nonparametric. Based on this method, parameters of copula are obtained.

Results: A clear advantage of the copula-based model is that it allows for maximum-likelihood estimation using all available data.

Conclusion: The aim of the method is basic to find the parameters that make the likelihood functions get its maximum value. With the help of the maximum-likelihood estimation method, for copula families obtain likelihood values. This values, Akaike information criteria (AIC) and Schwartz information criteria (SIC) are used to determine which copula supplies to suitability to the data set.
\end{abstract}

Key Words: Copula Function, GARCH method, Kendall Tau, Spearman Rho, Akaike information criteria, Schwartz information criteria.

\section{Introduction}

In the past years, the standard method of estimating dependence has been Pearson's correlation coefficient, which is based on the multivariate Gaussian distribution. However, as Fama (1963) noted, financial time series do not provide the assumption of normality. Embrechts, McNeil, and Straumann (1999) proved that Pearson's correlation coefficient is not sufficient to show the dependence between variables not belonging to the family of elliptical distributions. That is to say there was a need for the establishment of new methods to overcome the drawbacks of Person's correlation coefficient. Multivariate GARCH models formed such a status. The aim of this model is modeling of the conditional covariance and conditional correlation matrix.
Today, a commonly used second alternative can be found in the so called copulas introduced by Sklar (1959). The aim of this paper is to model GARCH for non-normal multivariate distributions using copulas. Copulas are defined functions that join one dimensional distribution functions together to form multivariate distribution functions by Sklar (1959).

There were very few practical applications of copulas. Nelsen (1999) gave definition of copula with mathematic perspective. Later applications of copulas were defined in finance Embrechts, P., A. McNeil and D. Straumann (2002).

Received: 30-03-2017 Accepted 26-04-2017 Available Online: 30-06-2017

1 Bitlis Eren University, Faculty of Science, Dept. of Statistics, Bitlis, TR

* Corresponding Author: Ayse Metin Karakas, E-mail: aysekarakas5767@gmail.com Phone: +90 4342220000 


\section{Material and Methods}

\subsection{GARCH Model}

GARCH model was first founded by generalizing ARCH model by Bollerslev and Eagle (1986).The GARCH $(\mathrm{p}, \mathrm{q})$ includes $\mathrm{p}$ lags of the variances in the linear $\mathrm{ARCH}(\mathrm{q})$ conditional variance equation. The variance equation can be generalized

$$
\sigma_{t}^{2}=w+\sum_{j=1}^{q} \alpha_{j} \varepsilon_{t-j}^{2}+\sum_{i=1}^{p} \beta_{i} \sigma_{t-i}^{2}
$$

Another extension is the Generalized ARCH or GARCH model. The GARCH model adds lags of the variance, ht-p, to the standard ARCH. A GARCH $(1,1)$ method refers to the presence of a first-order autoregressive ARCH statement and a first-order moving average GARCH statement. For GARCH $(\mathrm{p}, \mathrm{q})$

$\varepsilon_{t}$ is the error terms from the mean the equation. $\varepsilon_{t}=\sigma_{t} Z_{t}$, here, $Z_{t}$ is separate stochastic piece and also $Z_{t}$ is residual series, $Z_{t}$ have zero mean identical and independent distribution, $\sigma_{t}$ is a time dependent standard deviation.

$$
\begin{aligned}
& \checkmark \quad \beta_{i} \geq 0, \alpha_{j} \geq 0 \text { and } \sum_{i=1}^{p} \beta_{i}+\sum_{j=1}^{q} \alpha_{j}<1 . \\
& \checkmark \quad \sum_{i=1}^{p} \beta_{i} \sigma_{t-i}^{2} \text { is show GARCH statements, } \sum_{j=1}^{q} \alpha_{j} \sigma_{t-j}^{2} \text { is show ARCH statements. }
\end{aligned}
$$

The parameter of ARCH statements and GARCH statements submit the influence of ARCH effect (past innovation) and GARCH effect on the conditional variance. The rate of this effect to the coming periods respectively.

\subsection{Copula Theory}

$$
\text { In general GARCH }(1,1) \text { is enough to use for this series }[3,7,18] \text {. }
$$

The copula is defined as a $C:[0,1]^{2} \rightarrow[0,1]$ that ensures the limiting conditions

$$
\begin{array}{ll}
\checkmark & C(u, 0)=C(0, u)=0 \text { and } C(u, 1)=C(1, u)=u, \forall u \in[0,1] . \\
\checkmark & \left(u_{1}, u_{2}, v_{1}, v_{2}\right) \in[0,1]^{4}, \text { such that } u_{1} \leq u_{2}, v_{1} \leq v_{2} \\
& C\left(u_{2}, v_{2}\right)-C\left(u_{2}, v_{1}\right)-C\left(u_{1}, v_{2}\right)+C\left(u_{1}, v_{1}\right) \geq 0 .
\end{array}
$$

Ultimately, for twice differentiable and 2-increasing property can be replaced by the condition

$$
c(u, v)=\frac{\partial^{2} C(u, v)}{\partial u \partial v} \geq 0
$$

where $c(u, v)$ is the copula density. In the following, for $n$-uniform random $U_{1}, U_{2}, \ldots, U_{n}$ variables, the joint distribution function $C$ is defined

$$
C\left(u_{1}, u_{2}, \ldots, u_{n}, \theta\right)=P\left(U_{1} \leq u_{1}, U_{2} \leq u_{2}, \ldots U_{n} \leq u_{n}\right) \text {. }
$$

Here $\theta$ is dependence parameter $[1,2,3,4,5,8,9,10,11]$.

\subsubsection{Sklar Theorem}

Let $X$ and $Y$ be random variables with continuous distribution functions $F_{X}$ and $F_{Y}$, with $F_{X}(X)$ and $F_{Y}(Y)$ are uniformly distributed on the interval $[0,1]$. Then, there is a copula such that for all $x, y \in R$,

$$
F_{X Y}(X, Y)=C\left(F_{X}(X), F_{Y}(Y)\right. \text {. }
$$

The copula $C$ for $(X, Y)$ is the joint distribution function for the pair $F_{X}(X), F_{Y}(Y)$ provided $F_{X}$ and $F_{Y}$ continuous $[1,2,3,4,6,9,11,12,13,14,15,16,17,20,21,22,23]$. 


\subsubsection{Archimedean Copula}

Let $\varphi$ define a function $\phi:[0,1] \rightarrow[0, \infty]$ which is continuous and provides:

$\checkmark \phi(1)=0, \phi(0)=\infty$.

$\checkmark$ For all $t \in(0,1), \phi^{\prime}(t)<0, \varphi$ is decreasing, for all $t \in(0,1) \varphi^{\prime \prime}(t) \geq 0, \varphi$ is convex.

$\varphi$ has an inverse $\varphi^{-1}:[0, \infty] \rightarrow[0,1]$, which has the same properties out of $\phi^{(-1)}(0)=1$ and $\phi^{(-1)}(\infty)=0$. The Archimedean Copula is defined by

$$
C(u, v)=\phi^{(-1)}[\phi(u)+\phi(v)] .
$$

$[10,12,16,19]$

\subsubsection{Gumbel Copula}

This Archimedean copula is defines with the help of generator function $\phi(t)=(-\ln t)^{\theta}, \theta \geq 1$;

$$
C_{\theta}(u, v)=\exp \left(-\left[(-\ln u)^{\theta}+(-\ln v)^{\theta}\right]^{1 / \theta}\right)
$$

where $\theta$ is the copula parameter restricted to $[1, \infty)$. This copula is asymmetric, with more weight in the right tail. Beside this, it is extreme value copula [12].

\subsubsection{Clayton Copula}

This Archimedean copula is defines with the help of generator function $\phi(t)=\frac{t^{-\theta}-1}{\theta}$,

$$
C_{\theta}(u, v)=\left(u^{-\theta}+v^{-\theta}-1\right) .
$$

where $\theta$ is the copula parameter restricted to $(0, \infty)$. This copula is also asymmetric, but with more weight in the left tail [13].

\subsubsection{Frank Copula}

This Archimedean copula is defines with the help of generator function; $\phi(t)=-\ln \frac{-e^{-\theta t}-1}{e^{-\theta}-1}$;

$$
C_{\theta}(u, v)=-\frac{1}{\theta} \ln \left(1+\frac{\left(e^{-\theta u}-1\right)\left(e^{-\theta v}-1\right)}{\left(e^{-\theta}-1\right)}\right)
$$

where $\theta$ is the copula parameter restricted to $(0, \infty)[13]$.

\subsubsection{Joe Copula}

This Archimedean copula is defines with the help of generator function; $\varphi(t)=-\ln \left[1-(1-t)^{\theta}\right]$

$$
C_{\theta}(u, v)=1-\left[(1-u)^{\theta}+(1-v)^{\theta}-\left((1-u)^{\theta}(1-v)^{\theta}\right]^{1 / \theta}\right.
$$

where $\theta$ is the copula parameter restricted to $[1, \infty]$. This copula family is similar to the Gumbel. The right tail positive dependence is stronger more than Gumbel [20]. 


\subsubsection{Plackett Copula}

This copula function is defines

$$
C(u, v)=\frac{1+(\theta-1)-\sqrt{[1+(\theta-1)(u+v)]^{2}-4 \theta(\theta-1) u v}}{2(\theta-1)} .
$$

Where $\theta$ is the copula parameter restricted to $(0, \infty)[20]$.

\subsubsection{Ali Mikhail Haq Copula}

This Archimedean copula is defines with the help of generator function $\varphi(t)=\ln [1-\theta(1-t)] / t$

$$
C_{\theta}(u, v)=\frac{u v}{1-\theta(1-u)(1-v)}
$$

where $\theta$ is the copula parameter restricted to $[-1,1][18]$.

\subsection{Measuring Dependence}

\subsubsection{Spearman Rho}

Similar to approach of Pearson correlation coefficient, to compute the correlation between the pairs $\left(R_{i}, S_{i}\right)$ of ranks have been used. Thus, Spearman's Rho

$$
\begin{gathered}
\rho_{n}=\frac{\sum_{i=1}^{n}\left(R_{i}-\bar{R}\right)\left(S_{i}-\bar{S}\right)}{\sqrt{\sum_{i=1}^{n}\left(R_{i}-\bar{R}\right)^{2} \sum_{i=1}^{n}\left(S_{i}-\bar{S}\right)^{2}}} \in[-1,1] \\
\bar{R}=\frac{1}{n} \sum_{i=1}^{n} R_{i}=\frac{n+1}{2}=\frac{1}{n} \sum_{i=1}^{n} S_{i}
\end{gathered}
$$

write. This coefficient that stated expediently in the form

$$
\rho_{n}=\frac{12}{n(n+1)(n-1)} \sum_{i=1}^{n} R_{i} S_{i}-3 \frac{n+1}{n-1} .
$$

Also, $\rho_{n}$ is asymptotically unbiased estimator of

$$
\rho=12 \int_{[0,1]^{2}} u v d C(u, v)-3=12 \int_{[0,1]^{2}} C(u, v) d u d v-3
$$

where the second equality is obtain. This statement extended;

$$
12 \int_{[0,1]^{2}} u v d C_{n}(u, v)-3=\frac{12}{n} \sum_{i=1}^{n} \frac{R_{i}}{n+1} \frac{S_{i}}{n+1}-3=\frac{n-1}{n+1} \rho_{n}
$$

and $C_{n} \rightarrow C$ as $n \rightarrow \infty$. Here the null hypothesis $H_{0}=C=\Pi$ of independence of $X$ and $Y$, the distribution of $\rho_{n}$ is normal with zero mean and variance $1 /(n-1)$, thus for $H_{0}$ approximate $\alpha=0.05$, $\sqrt{n-1}\left|\rho_{n}\right|>z_{\alpha / 2}=1,96[12,13,14,15]$. 


\subsubsection{Kendall Tau}

Another measure of dependence is Kendall Tau. This measure based on ranks given by

$$
\tau_{n}=\frac{P_{n}-Q_{n}}{\left(\begin{array}{l}
n \\
2
\end{array}\right)}=\frac{4}{n(n-1)} P_{n}-1
$$

where $P_{n}$ and $Q_{n}$ number of concordant and discordant pairs respectively. Here, $\left(X_{i}, Y_{i}\right),\left(X_{j}, Y_{j}\right)$ pairs are concordant $\left(X_{i}-X_{j}\right)\left(Y_{i}-Y_{j}\right)>0$ and these are $\operatorname{disconcordant}\left(X_{i}-X_{j}\right)\left(Y_{i}-Y_{j}\right)<0$. If $\left(X_{i}-X_{j}\right)\left(Y_{i}-Y_{j}\right)>0$; we can say $\left(R_{i}-R_{j}\right)\left(S_{i}-S_{j}\right)>0 . \tau_{n}$ is function of copula $C_{n}$. As $n \rightarrow \infty$, $C_{n} \rightarrow C, W=\frac{1}{n} \sum_{j=1}^{n} I_{i j}=\frac{1}{n} \#\left\{j: X_{j} \leq X_{i}, Y_{j} \leq Y_{i}\right\}$,

$$
\tau_{n}=4 \frac{n}{n-1} \bar{W}-\frac{n+3}{n-1}=4 \int_{[0,1]^{2}} C(u, v) d C(u, v)-1
$$

written. $\tau_{n}$ is asymptotically unbiased estimator of $\tau$ and $\tau_{n}$ is normal with zero mean and variance $2(2 n+5) /\{9 n(n-1)\}$. Here the null hypothesis $H_{0}=C=\Pi$ of independence of $X$ and $Y$, thus for $H_{0}$ approximate $\alpha=0.05, \sqrt{9 n(n-1) / 2(2 n+5)}\left|\tau_{n}\right|>1.96[12,13,14,15,22]$.

\begin{tabular}{|c|c|c|c|c|}
\hline Family & Generator & Parameter & Kendall Tau & Spearman Rho \\
\hline Gumbel & $\phi(t)=(-\ln t)^{\theta}$ & $\theta \in[1, \infty)$ & $\frac{\theta-1}{\theta}$ & \\
\hline Clayton & $\phi(t)=\frac{t^{-\theta}-1}{\theta}$ & $\theta \in[0, \infty)$ & $\frac{\theta}{\theta+2}$ & \\
\hline Frank & $\phi(t)=-\ln \frac{-e^{-\theta t}-1}{e^{-\theta}-1}$ & $\theta \in(-\infty, \infty)$ & $1-\frac{4}{\theta}\left[1-D_{1}(\theta)\right]$ & $1-\frac{12}{\theta}\left[D_{2}(-\theta)-D_{1}(-\theta)\right]$ \\
\hline Joe & $\varphi(t)=-\ln \left[1-(1-t)^{\theta}\right.$ & $\theta \in[1, \infty)$ & $1+\frac{4}{\theta} D_{J}(\theta)$ & - \\
\hline \multirow[t]{2}{*}{ Placket } & - & $\theta \in(0, \infty)$ & - & $\theta+1 \quad 2 \theta \ln \theta$ \\
\hline & & & & $\overline{\theta-1}-\overline{(\theta-1)^{2}}$ \\
\hline
\end{tabular}

Table 1: Generator, Parameter space, Kendall Tau and Spearman Rho values of Special Copula Families

\subsection{Copula estimation}

\subsubsection{Maximum Likelihood Method (MLE)}

Maximum likelihood method is the most used for copula. The aim of the method is basic to find the parameters that make the likelihood functions get its maximum value. It is given

$$
\begin{gathered}
f\left(x_{1}, x_{2}, \ldots, x_{n}\right)=c\left(F_{1}\left(x_{1}\right), F_{2}\left(x_{2}\right), \ldots, F_{n}\left(x_{n}\right)\right) \prod_{j=1}^{n} f_{j}\left(x_{j}\right) \\
c\left(F_{1}\left(x_{1}\right), F_{2}\left(x_{2}\right), \ldots, F_{n}\left(x_{n}\right)\right)=\frac{\partial^{n} c\left(F_{1}\left(x_{1}\right), F_{2}\left(x_{2}\right), \ldots, F_{n}\left(x_{n}\right)\right)}{\partial F_{1}\left(x_{1}\right), F_{2}\left(x_{2}\right), \ldots, F_{n}\left(x_{n}\right)} .
\end{gathered}
$$

Let $\left\{x_{1 t}, x_{2 t}, \ldots, x_{n t}\right\}_{t=1}^{T}$ is the sample data matrix, the likelihood functions can be given

$$
l(\theta)=\sum_{t=1}^{T} \ln \left(c\left(F_{1}\left(x_{1 t}\right), F_{2}\left(x_{2 t}\right), \ldots, F_{n}\left(x_{n t}\right)\right)+\sum_{t=1}^{T} \sum_{j=1}^{n} \ln f_{j}\left(x_{j t}\right) .\right.
$$

Natural Science and Discovery 2017; 3(2):13-24 
Accordingly, the maximum likelihood estimator is

$$
\hat{\theta}_{M L E}=\max _{\theta} l(\theta) .
$$

\subsubsection{Inference for marginal (IFM)}

This method is used to overcome the drawbacks of full maximum likelihood function. The aim of copula theory is separate between the univariate margins and the dependence structure. From equation (19)

$$
l(\theta)=\sum_{t=1}^{T} \ln \left(c\left(F_{1}\left(x_{1 t}, \theta_{1}\right), F_{2}\left(x_{2 t}, \theta_{2}\right), \ldots, F_{n}\left(x_{n t}, \theta_{n}\right), \alpha\right)+\sum_{t=1}^{T} \sum_{j=1}^{n} \ln f_{j}\left(x_{j t}, \theta_{j}\right)\right.
$$

write. In this equation (19) the vector of the parameters for the univariate marginal $\theta=\left(\theta_{1}, \theta_{2}, \ldots, \theta_{n}\right)$ and $\alpha$ is vector the parameters of copula. Accordingly, the fundamental idea of inference for margins is that it is forecasts the parameters for marginal distributions and copula separately in two stages.

$\checkmark$ Estimate the parameters $\theta_{j}$ from marginal distributions,

$$
\hat{\theta}_{j}=\underset{\theta_{t}}{\arg \max } \sum_{t=1}^{T} \ln f_{j}\left(x_{j t} ; \theta_{j}\right)
$$

$\checkmark$ Estimation of the vector of the copula parameters $\alpha$, used the $\hat{\theta}=\left(\hat{\theta}_{1}, \hat{\theta}_{2}, \ldots, \hat{\theta}_{n}\right)$;

$$
\hat{\alpha}_{I F M}=\underset{\alpha}{\arg \max } \sum_{t=1}^{T} \ln \left(c\left(F_{1}\left(x_{1 t}, \hat{\theta}_{1}\right), F_{2}\left(x_{2 t}, \hat{\theta}_{2}\right), \ldots, F_{n}\left(x_{n t}, \hat{\theta}_{n}\right) ; \alpha\right)\right.
$$

\subsection{Tail Dependence of Copulas}

$$
[12,13,14,15] \text {. }
$$

In order to estimate the copula from bivariate observational data sets, we use the tail dependence concept. It relates the amount of dependence in the upper-right quadrant tail or in the lower-left-quadrant tail of a bivariate distribution. The upper and lower tail dependence parameters; If a bivariate copula $\mathrm{C}$ is such that; it is that upper tail dependence written,

$$
\lambda_{U}=\lim _{v \rightarrow 1} \frac{1-2 v+C(v, v)}{(1-v)}
$$

\begin{tabular}{|c|c|c|}
\hline $\begin{array}{l}\text { Copula } \\
\text { Family }\end{array}$ & $\lambda_{U}$ & $\lambda_{L}$ \\
\hline Gumbel & $2-2^{1 / \theta}$ & 0 \\
\hline $\begin{array}{c}\text { Joe } \\
\text { Copula }\end{array}$ & $2-2^{1 / \theta}$ & 0 \\
\hline $\begin{array}{c}\text { AMH } \\
\text { Copula }\end{array}$ & 0 & $= \begin{cases}\lim _{v \rightarrow 0} v / 1-\theta(1-v)^{2}=0,5 & \text { for } \theta=1 \\
\lim _{v \rightarrow 0} v / 1-\theta(1-v)^{2}=0,5 & \text { for } \theta<0\end{cases}$ \\
\hline $\begin{array}{l}\text { Clayton } \\
\text { Copula }\end{array}$ & 0 & $2^{-1 / \theta}$ \\
\hline $\begin{array}{c}\text { Frank } \\
\text { Copula }\end{array}$ & 0 & 0 \\
\hline $\begin{array}{c}\text { Plackett } \\
\text { Copula }\end{array}$ & 0 & 0 \\
\hline
\end{tabular}

Similarly, lower tail dependence is written;

$$
\lambda_{L}=\lim _{v \rightarrow 0} \frac{C(v, v)}{v} .
$$

Table 2: For copula familes upper and lower tail dependence 


\subsection{Copula-GARCH Estimation}

There are some approaches to model dependence. Many researchers prefer multivariate normal and t distribution to model in applications and GARCH model is widely used in this application. So, we prefer copula instead of multivariate GARCH to model dependence. The most important feature of copula is not requiring any assumptions of the margins normal distribution. Beside this, copula permit to separate a high dimensional joint distribution into its marginal distributions and copula function use to link them together. For GARCH model, there are many parameters which estimation more difficult. Compare to multivariate GARCH models and other multivariate models, copula is more suitable to model dependence structure. For the series, to model dependence structure, other selection criteria are Akaike's information criterion (AIC) and Schwarz's criterion (SIC). These;

$$
\begin{aligned}
A I C & =-2 \log L+2 k / n \\
S I C & =-2 \log L+k \ln (n) / n .
\end{aligned}
$$

Here, $\mathrm{k}$ is the number of estimated parameter for each model, $\mathrm{n}$ size of sample $[3,19]$.

\section{Application}

\subsection{Data Description}

In this study, I used data set (X, Y, Z, T) which generated from Weilbull distribution. I define the log-returns of series. Table 3 and Table 4 contain respectively descriptive statistics of $X, Y, Z, T$ series and $X, Y, Z, T$ return series. As submitted in these results, the means of $\mathrm{X}, \mathrm{Y}, \mathrm{Z}, \mathrm{T}$ series are not nearby to zero and standard deviations are a little bit. The Skewness means that X, Y, Z, T series are positive. The Kurtosis of X, Y, Z, T series are positive. The meaning of positive skewness is that $\mathrm{X}, \mathrm{Y}, \mathrm{Z}, \mathrm{T}$ series have the longer right tail of density.

Table 3: Descriptive statistics of $\mathrm{X}, \mathrm{Y}, \mathrm{Z}, \mathrm{T}$ series

\begin{tabular}{ccccc}
\hline & $\mathrm{X}$ & $\mathrm{Y}$ & $\mathrm{Z}$ & $\mathrm{T}$ \\
\hline mean & 1,041126 & 0,939809 & 0,991116 & 1,059747 \\
median & 0,747425 & 0,615185 & 0,670895 & 0,777685 \\
maximum & 6,726771 & 7,982274 & 8,028318 & 5,703414 \\
minumum & 0,000522 & 0,000671 & 0,005108 & 0,006594 \\
Std.dev. & 1,064711 & 0,963039 & 1,026534 & 0,967569 \\
Skewness & 2,205341 & 2,153105 & 2,438068 & 1,563574 \\
Kurtosis & 9,755806 & 11,01666 & 12,41151 & 5,763879 \\
Jarque-Bera & 1084,917 & 1380,170 & 1872,554 & 290,3015 \\
\hline
\end{tabular}
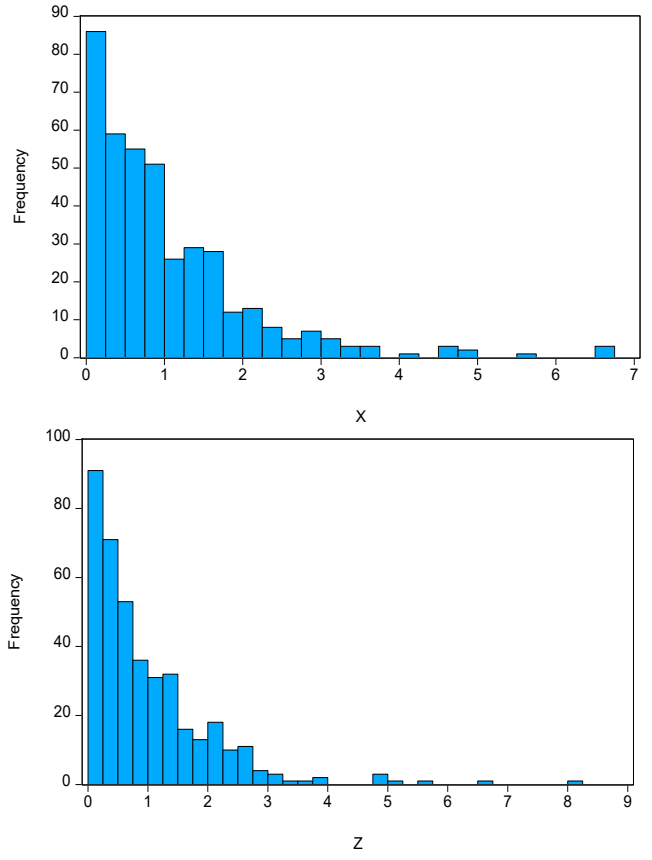

Figure 1: Frequency of $\mathrm{X}, \mathrm{Y}, \mathrm{Z}$ and $\mathrm{T}$ series
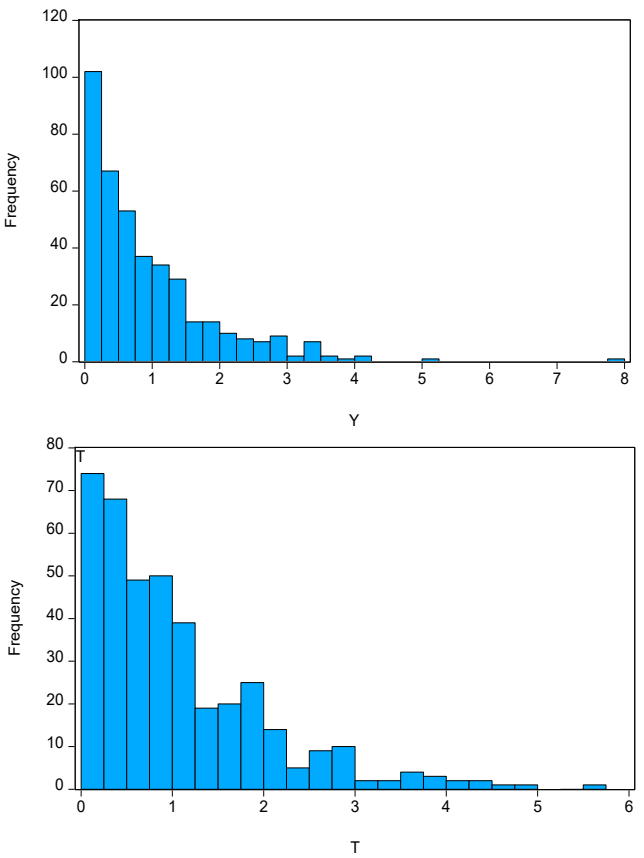

Natural Science and Discovery 2017; 3(2):13-24 
Table 4: Descriptive statistics of X,Y,Z,T return series

\begin{tabular}{ccccc}
\hline & $\mathrm{X}$ & $\mathrm{Y}$ & $\mathrm{Z}$ & $\mathrm{T}$ \\
\hline mean & $-0,007332$ & $-0,000379$ & 0,002451 & 0,001740 \\
median & 0,008150 & $-0,035048$ & $-0,008549$ & $-0,096574$ \\
maximum & 5,858241 & 7,341030 & 5,238800 & 5,813359 \\
minumum & $-6,040652$ & $-6,934251$ & $-5,860342$ & $-5,833584$ \\
Std.dev. & 1,765448 & 1,880493 & 1,707246 & 1,592205 \\
Skewness & 0,004231 & 0,155597 & 0,139830 & 0,187150 \\
Kurtosis & 3,739595 & 3,666246 & 3,727014 & 3,606463 \\
Jarque-Bera & 9,095071 & 8,989556 & 10,08736 & 8,443801 \\
\hline
\end{tabular}
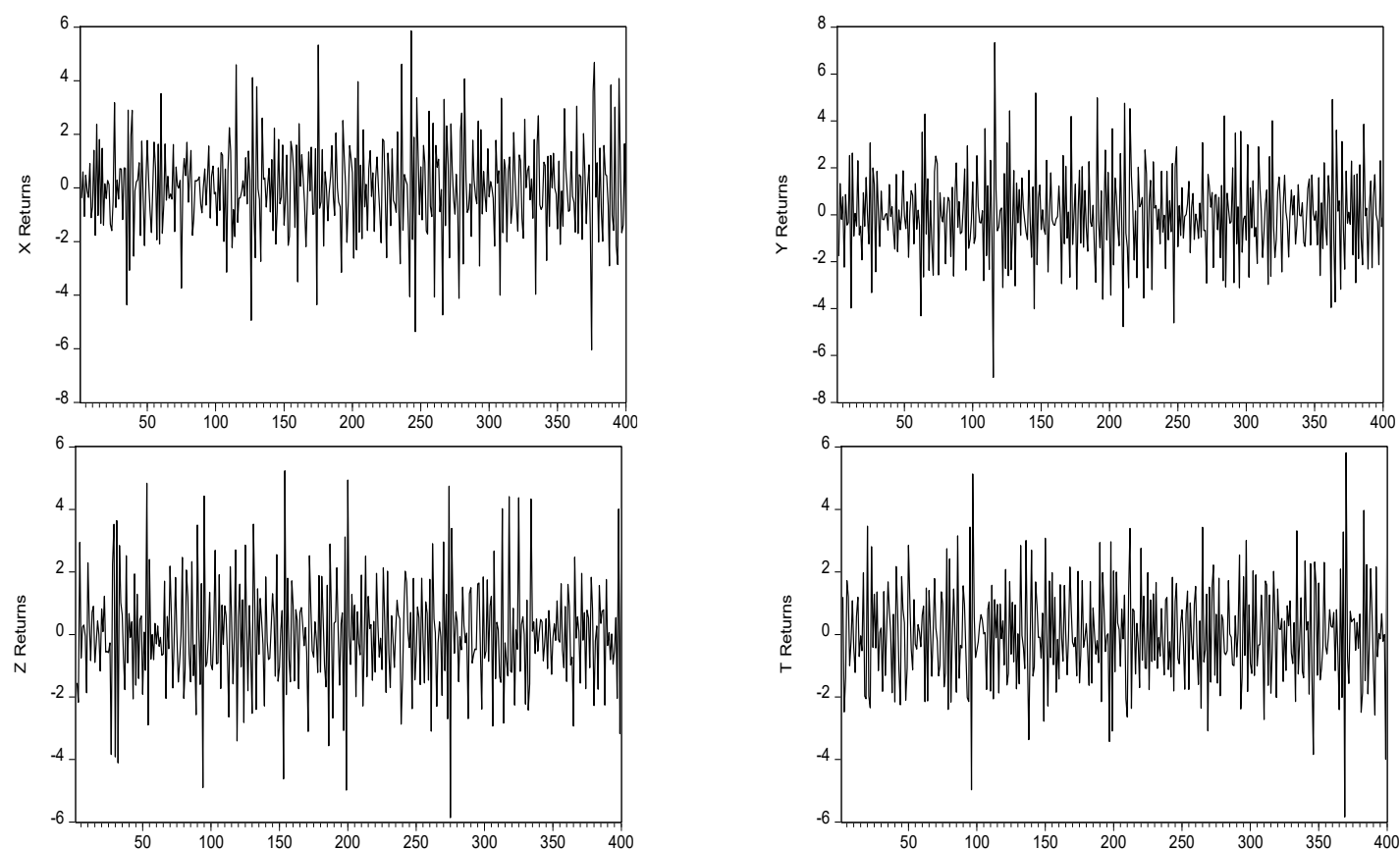

Figure 2: Returns of $\mathrm{X}, \mathrm{Y}, \mathrm{Z}$ and $\mathrm{T}$ series

\subsection{Modeling the marginal distribution}

In table 3,4,5,6, for $\mathrm{X}, \mathrm{Y}, \mathrm{Z}$ and $\mathrm{T}$ return series are given marginal modeling. In these tables, there are coefficients for variance equation. In the equation (1) $w$ is $\mathrm{C}, \alpha$ is $\mathrm{ARCH}$ (1) and $\beta$ is GARCH (1). Accordingly this equation, the sum of the ARCH and GARCH coefficients ( $\alpha+\beta<1)$ is very close to one, indicating that volatility shocks for this series are quite persistent. This result is often observed in high frequency data.

Table 5: X return Series Marginal Modeling

\begin{tabular}{ccc}
\hline & Gaussian & Standard Error \\
\hline C & 2,403845 & 0,382825 \\
ARCH $(1)$ & 0,397481 & 0,099624 \\
GARCH(1) & $-0,137874$ & 0,099005 \\
LogL & $-775,4711$ & - \\
AIC & 3,907123 & - \\
SIC & 3,947113 & - \\
\hline
\end{tabular}


Table 6: Y return Series Marginal Modeling

\begin{tabular}{ccc}
\hline & Gaussian & Standard Error \\
\hline C & 2,414059 & 0,519193 \\
ARCH $(1)$ & $-0,335428$ & 0,104123 \\
GARCH(1) & $-0,017985$ & 0,120611 \\
LogL & $-801,0585$ & - \\
AIC & 4,035381 & - \\
SIC & 4,075370 & - \\
\hline
\end{tabular}

Table 7: Z return Series Marginal Modeling

\begin{tabular}{ccc}
\hline & Gaussian & Standard Error \\
\hline C & 2,200512 & 0,349086 \\
ARCH(1) & 0,393349 & 0,104596 \\
GARCH(1) & $-0,155193$ & 0,110172 \\
LogL & $-754,4844$ & - \\
AIC & 3,801927 & - \\
SIC & 3,841916 & - \\
\hline
\end{tabular}

Table 8: T return Series Marginal Modeling

\begin{tabular}{ccc}
\hline & Gaussian & Standard Error \\
\hline C & 1,330661 & 0,372758 \\
ARCH(1) & 0,339488 & 0,100450 \\
GARCH(1) & 0,125565 & 0,180168 \\
LogL & $-724,8858$ & - \\
AIC & 3,678625 & - \\
SIC & 3,718615 & - \\
\hline
\end{tabular}

\subsection{With Copula Modeling of the Dependence Structure}

In this study, to model dependence, I present five copula families. I used to select Kendall's Tau and Spearman's Rho rank correlation statistics in our study, so the correlations parameters corresponding to each copula are obtained based on Kendall's Tau and Spearman's Rho. Maximum Likelihood Estimation method is used applied to estimation copula parameters. Accordingly, in table 11, 12, 13, 14, 15, 16 for copula families parameter values and Logl, AIC and SIC values is calculated. According to this values, with the help of equation, (19), (25) and (26), in table 11, relationship of X and Y series is positive weak relation and based on the AIC and SIC value we conclude that dependence structure of $\mathrm{X}$ and $\mathrm{Y}$ series is modeled by Ali Mikhail Haq copula ( $\theta=0,14722356)$, in table 12 relationship of $Z$ and $\mathrm{T}$ series is positive weak relation and based on the AIC and SIC value we conclude that dependence structure of $Z$ and $\mathrm{T}$ series is modeled by Clayton copula ( $\theta=0,0682523$ ), in table 13, relationship of $\mathrm{X}$ and $\mathrm{Z}$ series is negative weak relation based on the AIC and SIC value we conclude that dependence structure of $X$ and $Z$ series is modeled by Frank $(\theta=-0,108012)$, similarly in table 14 , in table 15 and in table 16 respectively, relationship of $\mathrm{X}$ and $\mathrm{T}$ series is negative weak relation and based on the AIC and SIC value we conclude that dependence structure of $\mathrm{X}$ and $\mathrm{T}$ series is modeled by Frank ( $\theta=-0,351430$ ), relationship of $\mathrm{Y}$ and $\mathrm{Z}$ series is positive weak relation and based on the AIC and SIC value we conclude that dependence structure of $\mathrm{Y}$ and $\mathrm{Z}$ series is modeled by Ali Mikhail Haq $(\theta=0,053274)$, relationship of $\mathrm{Y}$ and $\mathrm{T}$ series is negative weak relation and based on the AIC and SIC value we conclude that dependence structure of $\mathrm{Y}$ and $\mathrm{T}$ series is modeled by Frank $(\theta=-0,072003)$.

Table 9: For X, Y, Z, T series Kendall Tau $(\tau)$ rank correlation

\begin{tabular}{ccccc} 
& $\mathrm{X}$ & $\mathrm{Y}$ & $\mathrm{Z}$ & $\mathrm{T}$ \\
\hline $\mathrm{X}$ & 1 & 0,034 & $-0,012$ & $-0,039$ \\
$\mathrm{Y}$ & 0,034 & 1 & 0,012 & $-0,008$ \\
$\mathrm{Z}$ & $-0,012$ & 0,012 & 1 & 0,033 \\
$\mathrm{~T}$ & $-0,039$ & $-0,008$ & 0,033 & 1 \\
\hline
\end{tabular}


Table 10: For X, Y, Z, T series Spearman Rho $(\rho)$ rank correlation

$\begin{array}{ccccc} & \mathrm{X} & \mathrm{Y} & \mathrm{Z} & \mathrm{T} \\ \mathrm{X} & 1 & 0,05 & -0,019 & -0,059 \\ \mathrm{Y} & 0,05 & 1 & 0,015 & -0,013 \\ \mathrm{Z} & -0,019 & 0,015 & 1 & 0,049 \\ \mathrm{~T} & -0,059 & -0,013 & 0,049 & 1\end{array}$

Table 11: $\mathrm{X}$ and Y series Dependence Structure Modeling

\begin{tabular}{cccccc}
\hline Copula Family & $\theta$ & $\sigma$ & Logl & AIC & SIC \\
\hline Joe Copula & 1,061005 & 0,002373 & $-95,8729$ & 191,7558 & 191,7588 \\
AMH Copula & 0,1472356 & 0,0025 & 0,208139 & $-0,4066278$ & $-0,386320$ \\
Clayton & 0,070393 & 0,002246 & $-0,24994$ & 0,50988 & 0,51289 \\
Copula & 0,306286 & 0,002714 & $-59,874$ & 119,758 & 119,761 \\
Frank Copula & $0,032,822$ & 1265,654 & 1265,657 \\
$\begin{array}{c}\text { Plackett } \\
\text { Copula }\end{array}$ & 1,161695 & 0,002504 & $-632,822$ \\
\hline
\end{tabular}

Table 12: $Z$ and $T$ series Dependence Structure Modeling

\begin{tabular}{cccccc}
\hline Copula Family & $\theta$ & $\sigma$ & Logl & AIC & SIC \\
\hline Joe Copula & 1,059141 & 0,002368 & $-102,134$ & 204,278 & 204,281 \\
AMH Copula & 0,1430673 & 0,002501 & 0,226331 & $-0,44266$ & $-0,43965$ \\
Clayton & 0,0682523 & 0,002252 & 0,625233 & $-1,24047$ & $-1,23746$ \\
Copula & 0,2972623 & 0,002545 & $-57,9673$ & 115,9446 & 115,9476 \\
$\begin{array}{c}\text { Frank Copula } \\
\text { Plackett }\end{array}$ & 1,158477 & 0,004427 & $-639,803$ & 1279,616 & 1279,619 \\
$\quad$ Copula & & & & & \\
\hline
\end{tabular}

Table 13: $X$ and $Z$ series Dependence Structure Modeling

\begin{tabular}{|c|c|c|c|c|c|}
\hline Copula Family & $\theta$ & $\sigma$ & Logl & AIC & $\mathrm{SIC}$ \\
\hline Joe Copula & 0,979581 & 0,002351 & $-49,1176$ & 98,2452 & 98,24821 \\
\hline AMH Copula & $-0,054732$ & 0,002532 & 0,030216 & $-0,05043$ & $-0,04742$ \\
\hline $\begin{array}{l}\text { Clayton } \\
\text { Copula }\end{array}$ & $-0,023715$ & 0,002224 & 0,112861 & $-0,21572$ & $-0,21271$ \\
\hline Frank Copula & $-0,108012$ & 0,000331 & 17,68412 & $-35,3582$ & $-35,3552$ \\
\hline $\begin{array}{c}\text { Plackett } \\
\text { Copula }\end{array}$ & 0,9445883 & 0,0025 & $-1005,09$ & 2010,19 & 2010,193 \\
\hline
\end{tabular}

Table 14: $\mathrm{X}$ and $\mathrm{T}$ series Dependence Structure Modeling

\begin{tabular}{cccccc}
\hline Copula Family & $\theta$ & $\sigma$ & Logl & AIC & SIC \\
\hline Joe Copula & 0,9356073 & 0,002369 & $-25,5028$ & 51,0156 & 51,01861 \\
AMH Copula & $-0,183338$ & 0,002632 & 0,292312 & $-0,57462$ & $-0,57161$ \\
Clayton & $-0,075072$ & 0,002382 & 0,509709 & $-1,00942$ & $-1,00641$ \\
Copula & $-0,351430$ & 0,003511 & 48,15021 & $-96,2904$ & $-96,2874$ \\
$\begin{array}{c}\text { Frank Copula } \\
\text { Plackett }\end{array}$ & 0,837624 & 0,002504 & $-631,274$ & 1262,558 & 1262,561 \\
$\quad$ Copula & & & &
\end{tabular}

Natural Science and Discovery 2017; 3(2):13-24 
Table 15: $\mathrm{Y}$ and $\mathrm{Z}$ series Dependence Structure Modeling

\begin{tabular}{cccccc}
\hline Copula Family & $\theta$ & $\sigma$ & Logl & AIC & SIC \\
\hline Joe Copula & 1,020986 & 0,002353 & $-73,1704$ & 146,3508 & 146,3538 \\
AMH Copula & 0,053274 & 0,002503 & 0,015528 & $-0,02106$ & $-0,01805$ \\
Clayton & 0,024291 & 0,002295 & $-0,11522$ & 0,24044 & 0,24345 \\
$\quad$ Copula & 0,018012 & 0,000391 & $-19,7092$ & 39,4284 & 0,24345 \\
Frank Copula & 0,00251 & $-1069,54$ & 2139,09 & 2139,093 \\
$\begin{array}{c}\text { Plackett } \\
\text { Copula }\end{array}$ & 1,046031 & 0,00251 &
\end{tabular}

Table 16: $\mathrm{Y}$ and T series Dependence Structure Modeling

\begin{tabular}{cccccc}
\hline Copula Family & $\theta$ & $\sigma$ & Logl & AIC & SIC \\
\hline Joe Copula & 0,098325 & 0,002347 & $-53,7173$ & 107,4446 & 107,4476 \\
AMH Copula & $-0,036351$ & 0,002523 & 0,015474 & $-0,02095$ & $-0,01794$ \\
$\quad$ Clayton & 0,015873 & 0,001967 & $-0,01493$ & 0,03986 & 0,04287 \\
Copula & $-0,072003$ & 0,000148 & 12,03848 & $-24,067$ & $-24,0639$ \\
$\begin{array}{c}\text { Frank Copula } \\
\text { Plackett }\end{array}$ & 0,961748 & 0,002501 & $-1133,87$ & 2267,75 & 2267,753 \\
Copula & & & & & \\
\hline
\end{tabular}

\section{Conclusion}

In this paper, I based on investigate the structure of dependence between $\mathrm{X}, \mathrm{Y}, \mathrm{Z}$ and $\mathrm{T}$ which are generated Weilbull distribution. Thus I used Copula- GARCH approach. Primarily, I formed the marginal distribution using GARCH $(1,1)$ method with Gaussian distribution. From this observed results, X, Y, Z, and T series were to close each other and had high frequency data. Also, these series have a strong long-term persistence in the volatility. For dependency structure between $\mathrm{X}, \mathrm{Y}, \mathrm{Z}$, and $\mathrm{T}$ series, copula functions are used. The Copula is made up of six pairs that $(\mathrm{X}, \mathrm{Y}),(\mathrm{Z}, \mathrm{T}),(\mathrm{X}, \mathrm{Z}),(\mathrm{X}, \mathrm{T}),(\mathrm{Y}, \mathrm{Z})$ and $(\mathrm{Y}, \mathrm{T})$. The dependence of $(\mathrm{X}, \mathrm{Y})$ is modeled Ali Mikhail Haq copula with the parameter value of 0,1472356, Kendall Tau 0,034 and Spearman Rho 0,05, the dependence of $(\mathrm{Z}, \mathrm{T})$ is suitable copula Clayton copula with the parameter value of 0,0682523, Kendall Tau 0,033 and Spearman Rho 0,049, the dependence of $(X, Z)$ is best copula Frank copula with the parameter value of $-0,108012$, Kendall Tau -0,012 and Spearman Rho -0,019. Likewise, for the dependence of $(\mathrm{X}, \mathrm{T}),(\mathrm{Y}, \mathrm{Z})$ and $(\mathrm{Y}, \mathrm{T})$ are best copulas respectively, Frank copula with the parameter value of $-0,351430$, Kendall Tau $-0,039$ and Spearman Rho -0,059, Ali Mikhail Haq Copula with the parameter value of 0,053274, Kendall Tau 0,012 and Spearman Rho -0,015, Frank Copula with the parameter value of -0,072003, Kendall Tau -0,008 and Spearman Rho $-0,013$.

Conflict of interest: The authors declare they have no potential conflicts of interest with respect to the research, authorship, and/or publication of this article, and declare study has ethical permissions if required.

Acknowledgement: Contribution of Authors; AK Concept, Design and Data Analysis, Writing, Editing 


\section{References}

1. A. Sklar. Fonctions de Repartition a n Dimensions et Leurs Marges. Publications de I'Institut de Statistique de I'University de Paris. 1959; 8: 229-231.

2. B. Schweitzer, E.F Wolff. On nonparametric measures of dependence for random variables. Annals of Statistics. 1981; 9: 879-885.

3. Chan, N.-H., J. Chen, X. Chen, Y. Fan and L. Peng Statistical Inference for Multivariate Residual Copula of GARCH Models. Statistica Sinica. 2009; 19: 53-70.

4. Cherubini, U., Luciano, E. Value-at-Risk Trade-off and Capital Allocation with Copulas.Economic Notes. 2001; 30: 235-256.

5. Cyril Caillault, Dominique Guegan. Empirical Estimation of Tail Dependence Using Copulas. Application to Asian Markets. Quantitative Finance, Taylor \& Francis. 2005; 5 : 489 - 501.

6. Dong Hwan Oha, Andrew J. Pattonb. High-dimensiona copula-based distributions with mixed frequency data. Journal of Econometrics. 2016; 193: 349-366.

7. Eagle Robert F., Bollersev Tim. Modelling the persistence of conditional variance. Econometric Reviews, 1986;5(1): $1-50$

8. Embrechts, P., A. McNeil, and D. Straumann. Correlation: Pitfalls and Alternatives. ETH Zentrum. 1999.

9. Embrechts, P., A. McNeil and D. Straumann. Correlation and Dependence Properties in Risk Management: Properties and Pitfalls, in M. Dempster, ed., Risk Management: Value at Risk and Beyond. Cambridge University Press. 2002.

10. Eugene F. Fama. Mandelbrot and the Stable Paretian Hypotesıs. The Journal of Business, 1963; 36: 420-429.

11. E.W. Frees, E.A. Valdez. Understanding relationships using copulas. North American Actuarial Journal. 1998; 2:1-25.

12. Genest C., J. MacKay.V. The joy of copulas: bivariate distributions with uniform marginal. The American Statisticien. 1986; 40: 280-283.
13. Genest C. L.P. Rivest. Statistical inference procedures for bivariate Archimedean copulas. Journal of the American Statistical Association. 1993; 88: 1034-1043.

14. Genest, C., Favre, A.-C. Everything You Always Wanted to Know About Copula Modelling butWere Afraid to Ask. Journal of Hydrologic Engineering. 2006; 12: 347-368.

15. Genest, C., Gendron, M., Boudeau-Brien, M. The Advent of Copulas in Finance. The European Journal of Finance. 2009; 15: 609-618.

16. Malevergne, Y., Sornette, D. Testing the Gaussian Copula Hypothesis for Financial Assets Dependences. Quantitative Finance. 2003; 3: 231-250.

17. Metin A, Calık S. Copula Function and Application with Economic Data. Turkish Journal of Science and Technology. 2012; 7: 199-204.

18. Pranesh Kumar. Probability Distributions and Estimation of Ali-Mikhail-Haq Copula, Applied Mathematical Sciences. 2010; 4: 657-666.

19. Riadh Aloui, MohamedSafouaneBenAïssab, Relationship between oil, stock prices and exchange rates: $A$ vine copula based GARCH method, North American Journal of Economics and Finance 37 (2016), 458-471.

20. R. Nelsen. An Introduction to Copulas.Springer, Verlag. New York. 1999.

21. Rosenberg, J., Schuermann, T. A General Approach to Integrated Risk Management withSkewed, Fat-tailed Risks, Journal of Financial Economics. 2006; 79: 569-614.

22. Shih, J.H., Louis, T.A. Inferences on the Association Parameter in Copula Models for Bivariate Survival Data. Biometrics. 1995;5: 1384-1399.

23. Quesade-Molina, J.J. A generalization of an identity of Hoeffding and some applications, J.Ital.Stat.Soc. 1992: 3.

Copyright (C) 2016 The Author(s); This is an open-access article distributed under the terms of the Creative Commons Attribution License (http://creativecommons.org/licenses/by/4.0), which permits unrestricted use, distribution, and reproduction in any medium, provided the original work is properly cited. All Rights reserved by international journal of Natural Science and Discovery

Natural Science and Discovery 2017; 3(2):13-24 